\title{
On the use of velocity adaptation to outperform the motion planning with dynamics awareness in aerial long-reach manipulators with two arms
}

\author{
A. Caballero ${ }^{1}$, M. Bejar ${ }^{2}$ and A. Ollero ${ }^{1}$
}

\begin{abstract}
This paper presents a new approach for motion planning in cluttered environment of an aerial robotic system with two arms for long reach manipulation (ARS-LRM). The motion planning problem is addressed considering jointly the aerial platform and the dual arm in order to achieve wider operating conditions. Since there exists a strong dynamical coupling between the multirotor and the dual arm, safety in obstacle avoidance will be assured by introducing dynamics awareness (DA) in the operation of the planner. On the other hand, the limited manoeuvrability of the system emphasizes the importance of energy and time efficiency in the generated trajectories. Accordingly, a RRT*-based algorithm has been employed to guarantee their optimality. The joint exploration for the complete set of motion variables in the two ARS-LRM subsystems requires a significant computational effort which can be alleviated if only the position variables are considered in the search tree. In order to maintain the velocity as an additional exploration degree, a mechanism for Velocity Adaptation (VA) has been implemented. The resulting motion planning strategy has been validated satisfactorily through simulation in a realistic industrial scenario.
\end{abstract}

\section{INTRODUCTION}

Among the numerous applications in which unmanned aerial vehicles (UAVs) can be used, aerial manipulation is arousing much interest. Potential applications in this field include instrument deployment, maintenance operation and contact inspection in industrial sites in which the access is very dangerous or costly. The motivation is to decrease risks and operational costs. Small size rotorcraft can indeed access to hard-to-reach places more easily than human operators, avoiding unnecessary risks for industrial workers and allowing inspection and maintenance operations without shutdowns of the facilities (the mandatory safety policy in case of human operation) and without the use of scaffolding or cranes.

These new promising applications of aerial robotic systems for manipulation tasks bring also new challenges. On the one hand, it is necessary to develop new manipulation tools such as adapted arms or grippers that can be seamlessly integrated into the airframe. Furthermore, the existing algorithms for operating autonomously the UAV and the manipulators should be extended for the integrated system. In this respect, one of the most challenging issues is the development of new methods that consider both the UAV and

${ }^{1}$ A. Caballero (e-mail: alvarocaballero@us.es) and A. Ollero (e-mail: aollero@us.es) are with the University of Seville, Seville (Spain).

${ }^{2}$ M. Bejar (e-mail: mbejdomeupo.es) is with the University Pablo de Olavide, Seville (Spain). the manipulator dynamics when planning the motion of the complete system. When moving between different locations inside a dense industrial installation, this planning will be essential for the generation of accurate and collision-free movements close to obstacles.

Many research works about aerial manipulation have been recently published. [1] presents the design of several lightweight, low-complexity grippers that allow quadrotors to grasp and perch on branches or beams and pick up and transport payloads. In a very different system scale, [2] proposes a system for aerial manipulation, composed of a helicopter and an industrial manipulator.

However, among the different contributions focused on aerial manipulation, very few of them consider configurations with more than one arm. The need to employ several arms can be justified in tasks such as transportation of long pieces (to avoid swinging movements), application of torques or other manipulation tasks that require two hands simultaneously. Thus, [3] proposes a dual arm aerial manipulator to turn a valve that requires a tightly integrated control scheme between aircraft and both manipulators. On the other hand, in [4] a human-size and lightweight dual arm manipulator is integrated in a multirotor platform and tested in outdoor flights. Concerning theoretical contributions, [5] introduces a generic planar aerial manipulator with any number of arms attached at the center of mass of a UAV. The authors prove that this kind of systems are differentially flat regardless the number of joints of each arm and their kinematic and dynamic parameters.

On the other hand, although a large amount of works have been focused on the development of control techniques for the system integrating the aerial vehicle and the manipulator devices, not many of them deal with the associated motion planning problem. Furthermore, the existing contributions like [6] usually assume a strong simplification by addressing the planning problem in a decoupled way, i.e. adopting independent planners for the UAV and the manipulators that swap their operation according to the mission phase. In contrast to previous works, the authors of this paper presented in [7] a novel algorithm that considers jointly the aerial platform and the manipulators within the planning operation. This integrated strategy allows the consideration of wider and safer operating conditions.

Concerning motion planning problems where dynamics can not be neglected, there also exists some contributions aiming at finding admissible and collision-free trajectories 


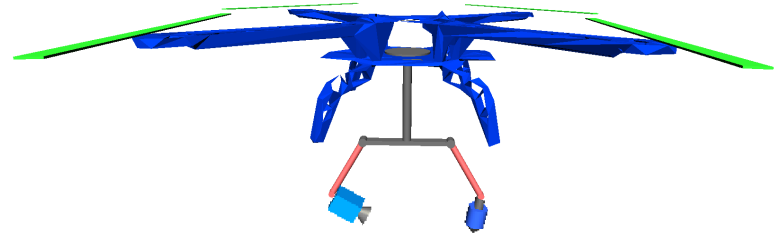

Fig. 1. Aerial robotic system with two arms for long-reach manipulation (ARS-LRM).

for such systems. In a relevant contribution, [8] proposes kinodynamic motion planners that consider kinematic and dynamic constraints during trajectory generation. However, these planners require a planning space twice the dimension of the configuration space -configuration variables together with their associated velocities-, which implies high computational costs. Alternatively, [9] and [10] propose other methods that split the motion planning problem into two stages. In the first stage a basic planner searches for a path compatible with a bounding sphere that replaces the system. In the second stage the path is transformed into a trajectory compatible with the kinodynamic constraints. A significant drawback of these methods comes from the approximation required in first stage since it could complicate the existence of a collision-free path. Along with these research lines, an extension of [7] was presented by the authors of this paper in [11], an enhanced version of the planning algorithm that introduces dynamic awareness within the operation of the planner for robust obstacle avoidance.

This paper extends the aforementioned contributions of the authors in [7] and [11]. The common basis of these works is the exploration of dual arm configurations that guarantee long-reach manipulation in those scenarios where the target is far from the operation area of the UAV. In order to meet these requirements a new aerial robotic system with two arms for long-reach manipulation (ARS-LRM) is proposed. More precisely, the system consists of a multirotor with a long bar extension that incorporates a lightweight dual arm in the tip (see Fig. 1). Thus, the long bar extension increases considerably the safety distance between rotors and manipulated objects while the dual arm offers extended manipulation capabilities.

The aerial platform and the dual arm device are considered jointly within the planner operation, which constitutes a remarkable difference to previous contributions where the planning problem was addressed in a decoupled way. This integrated strategy allows the consideration of a more complete set of system states that in turn will make it possible to achieve wider and safer operating conditions. The strong dynamical coupling between the multirotor and the dual arm makes necessary to introduce dynamics awareness (DA) in the planner for robust obstacle avoidance. Accordingly, the expansion of the search tree is based on the behaviour of the controlled system. Regarding the operation basis of the planner, an RRT*-based algorithm that optimizes energy performance has been developed.

The most relevant contribution of this paper is the im- plementation of a new Velocity Adaptation (VA) mechanism that complements the dynamics awareness (DA) approach, which results in the RRT*-DAVA motion planning algorithm presented in this work. The VA mechanism allows a better optimization of the execution time of the planned trajectories but without extending the planning space and hence minimising the computational burden. Recalling the joint exploration mentioned before for the complete set of motion variables in the two ARS-LRM subsystems, this approach requires a significant computational effort. The latter can be alleviated if only the position variables are considered in the search tree. In order to maintain the velocity as an additional exploration degree, a mechanism for Velocity Adaptation (VA) based on an bounded iterative process has been implemented. The resulting motion planning strategy has been validated satisfactorily through simulation in one of the realistic industrial scenarios considered in [11], the chimney repairing task.

Sec. II of this paper presents the structure of the integrated platform, the corresponding multi-body dynamical model and finally the distributed control approach derived for the system. Then, in Sec. III, the planning algorithm is explained with special attention to the new Velocity Adaptation (VA) mechanism proposed in this paper. In order to better illustrate its benefits, Sec. IV defines the realistic industrial scenario considered, a chimney repairing task. After presenting the complete system as well as the motivating scenario, Sec. V includes several simulations of the controlled ARS-LRM system when following the planned trajectories given by the RRT*-DA and the RRT*-DAVA algorithms. The latter illustrates how the RRT*-DAVA algorithm outperforms the results obtained with RRT*-DA. Finally, Sec. VI is devoted to conclusions and future work.

\section{THE AERIAL LONG-REACH MANIPULATOR}

\section{A. System Description}

As can be seen in Fig. 2, the proposed aerial robotic system for long-reach manipulation (ARS-LRM) consists of a multirotor with a long bar extension that incorporates a lightweight dual arm in the tip. This configuration allows aerial manipulation tasks in hard-to-reach places increasing considerably the safety distance between rotors and manip-

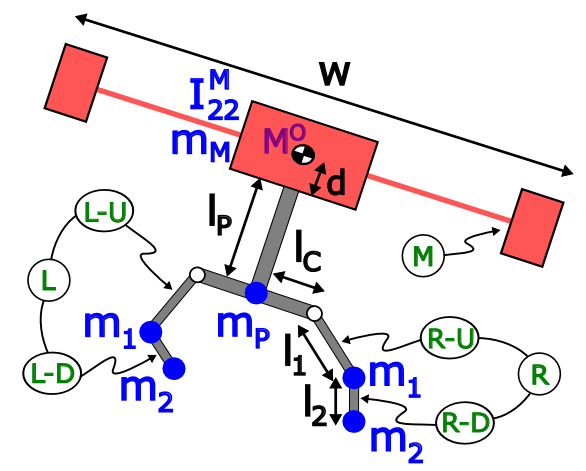

Fig. 2. Geometry and mass distribution of the ARS-LRM system. 
ulated objects. Furthermore, the dual arm offers extended manipulation capabilities with respect to the single arm configurations existing in the literature. In this first prototype of the system, each separate arm $L, R$ is composed of two links $L-U, L-D, R-U, R-D$ but further extensions of the manipulation chain are considered in future work.

A planar characterization of the system will serve for establishing a first proof of concept for the ARS-LRM setup. This simplified approach eases the modelling and control developments while maintaining the operation basis of the system. Following this assumption, the multirotor is characterized by a mass $m_{M}$, a principal moment of inertia $I_{22}^{M}$ and dimensions $2 d \times w$. Regarding the long bar, its longitude is given by $l_{P}$ and it is assumed to be aligned with the UAV center of mass $M^{O}$ at a distance $d$. The cross-piece in the tip is defined by a length of $2 l_{C}$. The total mass of the long bar and the cross-piece is $m_{P}$ and will be treated as a punctual mass located where the long bar and the crosspiece intersects for simplicity purposes. Finally, the two arms are characterized respectively by the lengths of their links $-l_{1}$ for upper links and $l_{2}$ for lower links- and their masses - $m_{1}$ and $m_{2^{-}}$, where again the masses will be treated as punctual masses located at the distal end of each link in order to derive more manageable expressions. The values of the aforementioned parameters are shown in Table I.

\section{B. Modelling}

According to [12], the dynamics of a multirotor is mostly determined by its mechanical model. This paper embraces the same assumption and consequently the behaviour of the ARS-LRM platform will be described by means of an elaborated mechanical model of the complete multi-body system. Kane's method has been used with this purpose since it holds some unique advantages when addressing multi-body robotic systems like the ARS-LRM. Of the latter, the most remarkable are the derivation of a compact model in first order differential equations that are uncoupled in the generalized speed derivatives as well as the easy computerization and the computational efficiency of the resulting equations of motion.

The configuration variables selected as system generalized coordinates are the longitudinal $q_{1}$ and vertical $q_{3}$ positions

TABLE I

ARS-LRM PARAMETERS

\begin{tabular}{cccc}
\hline & Parameter & Value & Units \\
\hline \hline Mass and Inertia & $m_{M}$ & 6.5 & $\mathrm{~kg}$ \\
\hline- & $I_{22}^{M}$ & 0.093 & $\mathrm{kgm}^{2}$ \\
\hline- & $m_{P}$ & 0.15 & $\mathrm{~kg}$ \\
\hline- & $m_{1}$ & 0.06 & $\mathrm{~kg}$ \\
\hline- & $m_{2}$ & 0.03 & $\mathrm{~kg}$ \\
\hline Geometry & $d$ & 0.1 & $\mathrm{~m}$ \\
\hline- & $w$ & 0.9 & $\mathrm{~m}$ \\
\hline- & $l_{P}$ & 0.2 & $\mathrm{~m}$ \\
\hline- & $l_{C}$ & 0.1 & $\mathrm{~m}$ \\
\hline- & $l_{1}$ & 0.15 & $\mathrm{~m}$ \\
\hline- & $l_{2}$ & 0.05 & $\mathrm{~m}$ \\
\hline
\end{tabular}

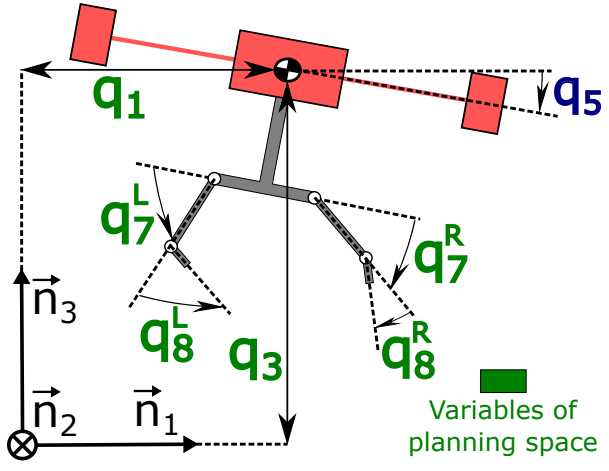

Fig. 3. Configuration variables of the ARS-LRM model. In green, the variables selected later for the planning space.

of the UAV center of mass $M^{O}$ in the inertial reference frame $N$, the multirotor pitch angle $q_{5}$ and the joint angles both for left $L$ and right $R$ arms $q_{7}^{L}, q_{8}^{L}, q_{7}^{R}$ and $q_{8}^{R}$ (see Fig. 3). Generalized speeds $u_{i}$ are defined as:

$$
\begin{aligned}
& { }^{M} \boldsymbol{\omega}^{R-U}=u_{7}^{R} \mathbf{n}_{2} \\
& { }^{N} \mathbf{v}^{M^{O}}=u_{1} \mathbf{n}_{1}+u_{3} \mathbf{n}_{\mathbf{3}} \quad{ }^{R-U} \boldsymbol{\omega}^{R-D}=u_{8}^{R} \mathbf{n}_{\mathbf{2}} \\
& { }^{N} \boldsymbol{\omega}^{M}=u_{5} \mathbf{n}_{\mathbf{2}} \\
& { }^{M} \boldsymbol{\omega}^{L-U}=-u_{7}^{L} \mathbf{n}_{\mathbf{2}} \\
& { }^{L-U} \boldsymbol{\omega}^{L-D}=-u_{8}^{L} \mathbf{n}_{\mathbf{2}}
\end{aligned}
$$

where ${ }^{N} \mathbf{v}^{M^{O}}$ is the velocity of the UAV center of mass $M^{O}$ with respect to the inertial reference frame $N$ and ${ }^{i} \boldsymbol{\omega}^{j}$ is the angular velocity of the element $j$ with respect to the element $i$ (see Fig. 2 to identify the different elements). Previous equations lead to the following kinematic differential equations:

$$
\begin{aligned}
& \dot{q}_{i}=u_{i}(i=1,3,5) \\
& \dot{q}_{j}^{k}=u_{j}^{k} \quad(j=7,8 ; k=R, L)
\end{aligned}
$$

Regarding forces and torques exerted on the ARS-LRM system (see Fig. 4), the rotors generate a resultant lifting force $F_{3} \mathbf{a}_{3}$ applied at the multirotor center of mass $M^{O}$ as well as a torque $T_{2} \mathbf{a}_{2}$ applied to rigid body $M$. On the other hand, control actions governing the manipulator are given by the torques applied to the arm joints $T_{7}^{R} \mathbf{a}_{2}, T_{8}^{R} \mathbf{a}_{2},-T_{7}^{L} \mathbf{a}_{2}$ and $-T_{8}^{L} \mathbf{a}_{2}$.

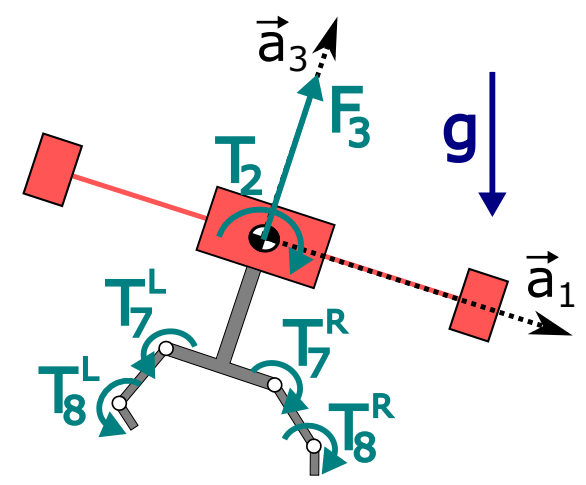

Fig. 4. Forces and torques applied to the ARS-LRM system. 
Application of Kane's method through MotionGenesis software [13] leads to the following dynamic differential equations for translation and rotation, where $\mathbf{A}, \mathbf{B}, \mathbf{C}$ and $\mathbf{D}$ are dense matrices depending on the configuration variables $q_{5}, q_{7}^{R}, q_{8}^{R}, q_{7}^{L}, q_{8}^{L}$ and the system parameters defined in Table I, and $g$ is the gravity acceleration.

$$
\left[\begin{array}{c}
\dot{u}_{1} \\
\dot{u}_{3} \\
\dot{u}_{5} \\
\dot{u}_{7}^{R} \\
\dot{u}_{8}^{R} \\
\dot{u}_{7}^{L} \\
\dot{u}_{8}^{L}
\end{array}\right]=\mathbf{A}\left[\begin{array}{c}
F_{3} \\
T_{2} \\
T_{7}^{R} \\
T_{8}^{R} \\
T_{7}^{L} \\
T_{8}^{L}
\end{array}\right]+\mathbf{B}\left[\begin{array}{c}
\left(u_{5}\right)^{2} \\
\left(u_{7}^{R}\right)^{2} \\
\left(u_{8}^{R}\right)^{2} \\
\left(u_{7}^{L}\right)^{2} \\
\left(u_{8}^{L}\right)^{2}
\end{array}\right]+\mathbf{C}\left[\begin{array}{c}
u_{5} u_{7}^{R} \\
u_{5} u_{8}^{R} \\
u_{5} u_{7}^{L} \\
u_{5} u_{8}^{L} \\
u_{7}^{R} u_{8}^{R} \\
u_{7}^{L} u_{8}^{L}
\end{array}\right]+\mathbf{D} g
$$

\section{Control}

After modelling the ARS-LRM system, a distributed control scheme (see Fig. 5) has been derived to provide the system with the capacity of executing navigation and manipulation manoeuvres. The objective is the completion of the simulation environment that will allow the investigation of new planning strategies for the ARS-LRM platform. A basic control structure that makes use of nonlinear control strategies based on model inversion shall suffice to complete the testbed.

Regarding the multirotor, the control scheme is inspired by [12] and consists in linearising the system through model inversion and applying PID control laws to the resultant dynamics. The underlying principle of control will be the adjustment of the multirotor lifting force vector, in order to generate the translational accelerations required to reduce position error. A general overview of the control scheme is shown in Fig. 6, where $D_{13}^{-1}, K_{5}^{-1}$ and $D_{5}^{-1}$ blocks represent, respectively, the inversions of the translational dynamics, rotational kinematics and rotational dynamics.

The control strategy selected for each arm is again based on linearisation through model inversion and PD control, which yields a nonlinear control law capable of commanding the link positions of both arms. The schematic representation of this approach is shown in Fig. 7 where $D_{78}^{-1}$ represents the block in charge of inverting arm dynamics and torques $T_{7}^{R}, T_{8}^{R}, T_{7}^{L}$ and $T_{8}^{L}$ correspond to the output signals of the controller.

The parameters of the controllers have been tuned by means of the classic pole assignment method. The selected

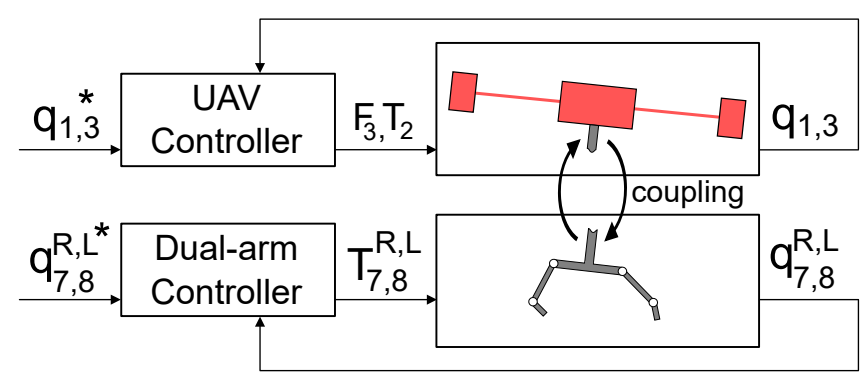

Fig. 5. Block diagram of the distributed control scheme.

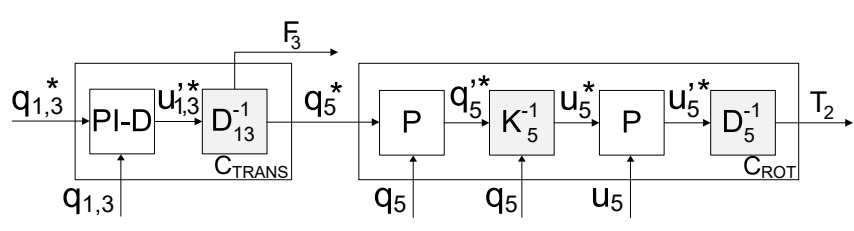

Fig. 6. Block diagram of the UAV controller.

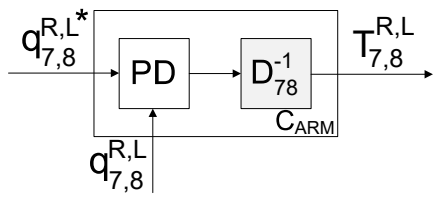

Fig. 7. Block diagram of the arm controller.

values constitute a trade-off that guarantees a proper dynamics range while the common mechanical limitations of this kind of systems are not overreached.

\section{MOTION PLANNER WITH DYNAMICS AWARENESS AND VELOCITY ADAPTATION}

\section{A. Fundamentals of Planner Operation}

The operation of the planner is based on an integrated exploration of the search space for both subsystems of the ARS-LRM. To this end, the complete set of configuration variables introduced in Sec. II-B for the aerial platform (with the exception of pitch angle $q_{5}$ ) and the dual arm device are considered jointly within the planning algorithm (see the variables in green colour in Fig. 3). This integrated strategy offers higher resolution in the planner operation since equivalent configurations in terms of final effector positions can be differentiated according to the positions of both the multirotor and the intermediate links. The latter makes it possible to achieve wider and safer operating conditions and constitutes a remarkable difference to previous contributions where the planning problem was addressed in a decoupled way.

Regarding the operation basis for the extension of the search tree, an RRT*-based algorithm that optimizes energy and time performance has been developed. Although the proposed algorithm poses certain structural similarities to the well-known RRT* approach, most of the intermediate functionalities have been customized to deal with the aerial manipulator under study. For instance, Fig. 8 illustrates how the two weighting parameters included in cost function $C F$ allow the prioritization of certain movements in the ARS-LRM system. The complete set of these specific derivations have been properly described in previous contribution [7].

\section{B. Dynamics Awareness}

The basic approach presented in previous subsection guarantee planned trajectories that are collision free as well as energy efficient. However, the strong dynamical coupling that exists in the ARS-LRM system between the multirotor and the dual arm requires further attention since it provokes 


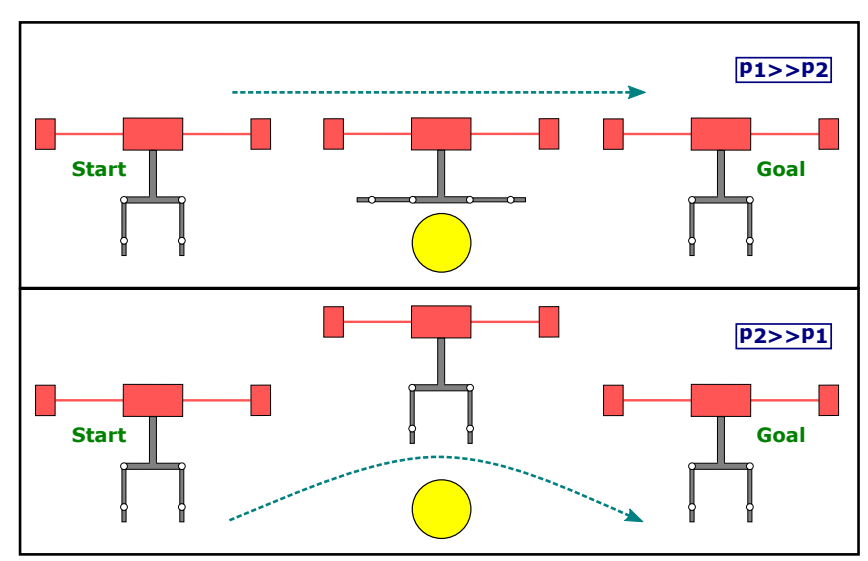

Fig. 8. Effect of the weighting parameters $p_{1,2}$ in the cost function $C F=$ $f\left(p_{1,2}\right) \cdot p_{1} \gg p_{2}$ prioritizes trajectories in which the UAV displacement is minimum while $p_{2} \gg p_{1}$ prioritizes the contrary.

considerable differences between planned and executed trajectories. The collision risk introduced by these differences is specially critical for cluttered environments like industrial sites. Fig. 9 illustrates an example of the undesired dynamic effects that must be avoided.

In order to overcome this undesired influence of the dynamical coupling, the inclusion of dynamics awareness (DA) in the planning algorithm is required. To this end, the expansion of the search tree is based on the behaviour of the controlled system, which means that collision detection is calculated through close-loop simulations of the controlled system (yellow solid line in Fig. 10) instead of using geometrical interpolation between states (green dashed line in Fig. 10). This guarantees that the resultant planned trajectories are both free of obstacles and compatible with the dynamic constraints of the ARS-LRM system. The approach being investigated for the dynamical analysis of the tree extension is based on a root-to-candidate validation. Thus, not only the dynamical feasibility of the new possible branch reaching the candidate node is analysed, but also the complete path from the tree root. For more details about this improvement of the basis algorithm, please refer to previous work of the authors [11].

\section{Velocity Adaptation}

This section presents the new Velocity Adaptation (VA) mechanism that complements the dynamics awareness (DA)

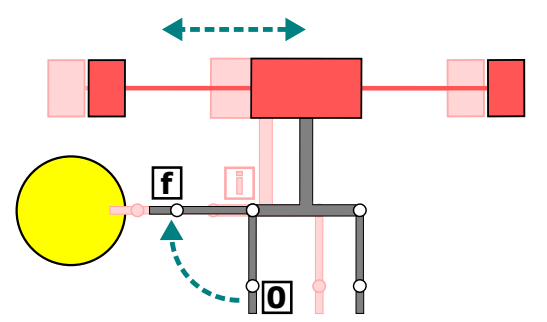

Fig. 9. ARS-LRM system spreading the left arm from initial position 0 to final position $f$. The UAV oscillation (shaded intermediate position $i$ ) produces a collision with the yellow obstacle.

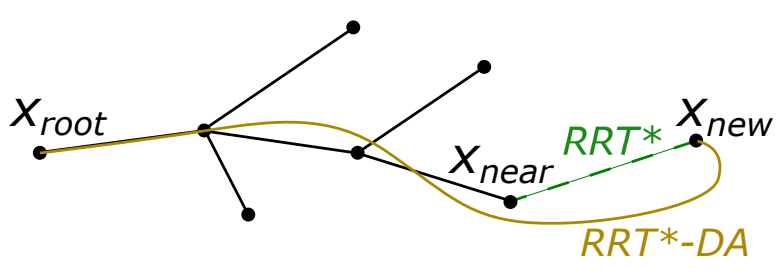

Fig. 10. Operation basis of the RRT*-DA algorithm.

approach. As mentioned in previous sections, the joint exploration for the two ARS-LRM subsystems requires a tradeoff between the desired completeness for the exploration and the associated computational cost. Previous developments did not consider velocity variations for optimizing the RRT*-based operation of the algorithm and hence they lead to constant velocity profiles in the resulting planned trajectories for both the aerial platform and the dual robotic arm. The performance of this approach is clearly non-optimal for non-homogeneously cluttered environments. In these cases the constant velocity profiles will end up equalling the lowest velocities required to safely avoid obstacles in the most cluttered areas.

In order to outperform the latter, the RRT*-DA algorithm is transformed into a more advanced algorithm that incorporates Velocity Adaptation at branch level during the whole operation of the planner (RRT*-DAVA). This Velocity Adaptation at branch level is implemented by means of an iterative process that is computationally bounded. In other words, only a discrete set of velocities are considered for each branch. In this way, the velocity optimisation reduces the expected execution time of the planned trajectory but without neither extending the planning space nor augmenting considerably the computational burden.

As mentioned before, the operation principle of the RRT*-DAVA algorithm is given by an iterative analysis of the maximum allowed velocity for each new branch in the search tree. More precisely, the velocity profile is initialised to the maximum value according to the mechanical constraints of the system and it is then decreased gradually when analysing the dynamics of the branch under study until a feasible behaviour is found. The fundamentals of the approach are illustrated in Fig. 11, where $u_{r e f}^{\max }, w_{\text {ref }}^{\max }$ are the initial maximum velocities due to mechanical constraints and $u_{r e f}, w_{r e f}$ correspond to the resulting maximum allowable

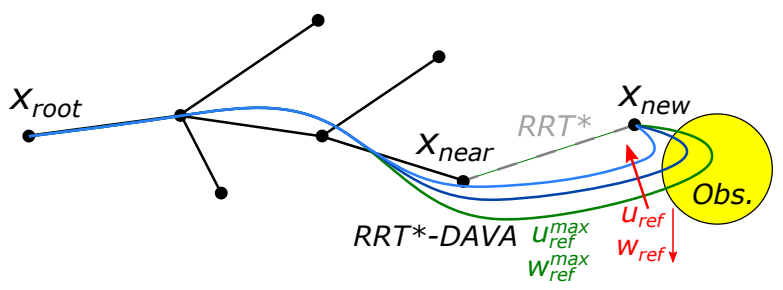

Fig. 11. Operation basis of the RRT*-DAVA algorithm. $u_{r e f}^{\max }, w_{\text {ref }}^{\max }$ are the initial maximum velocities and $u_{r e f}, w_{r e f}$ correspond to the resulting maximum allowable velocities that guarantee safe trajectories for the branch under study. 


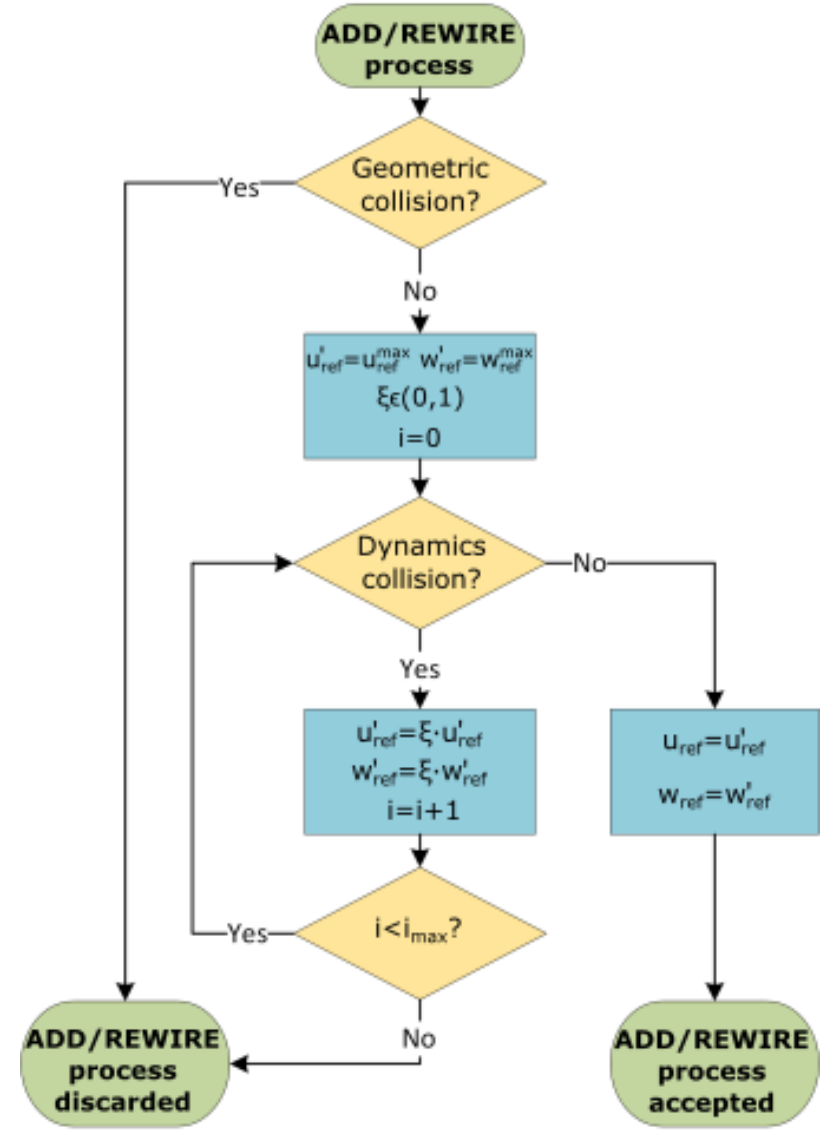

Fig. 12. Flow diagram representing the basis operation of the RRT*-DAVA algorithm.

velocities that guarantee safe trajectories for the branch under study.

Fig. 12 shows the detailed flow diagram corresponding to the VA approach. The Geometric collision block discards unavoidable collisions because of the geometrical path of the branch under study. With this purpose, in both the $A D D\left(x_{\text {nearest }}, x_{\text {near }}, x_{\text {new }}\right)$ and $\operatorname{REWIRE}\left(x_{\text {near }}, x_{\text {new }}\right)$ functions, collisions are firstly checked in the way described in Sec. Collision checking of [11]. Then, if there are not Geometric collisions, the Dynamics collision step analyses potential collisions motivated by the dynamical behaviour of the system (overshooting, ...) as described in Sec. Dynamics awareness of [11]. In the first place, it will consider the maximum reference velocities $u_{r e f}^{\max }, w_{r e f}^{\max }$. If any collision is detected, the reference velocities $v_{r e f}, w_{r e f}$ are reduced according to a scaling factor $\xi \in(0,1)$ and the Dynamics collision process is followed again until no collisions appears. As was advanced, the number of iterations is bounded with a limit of $i_{\max }$ that should be properly tuned to achieve a trade-off between the optimality of the solutions and the generated computational load.

\section{APPLICATION SCENARIO: CHIMNEY REPAIRING TASK}

In order to demonstrate the validity of the motion planning strategy presented in previous sections, the RRT*-DAVA

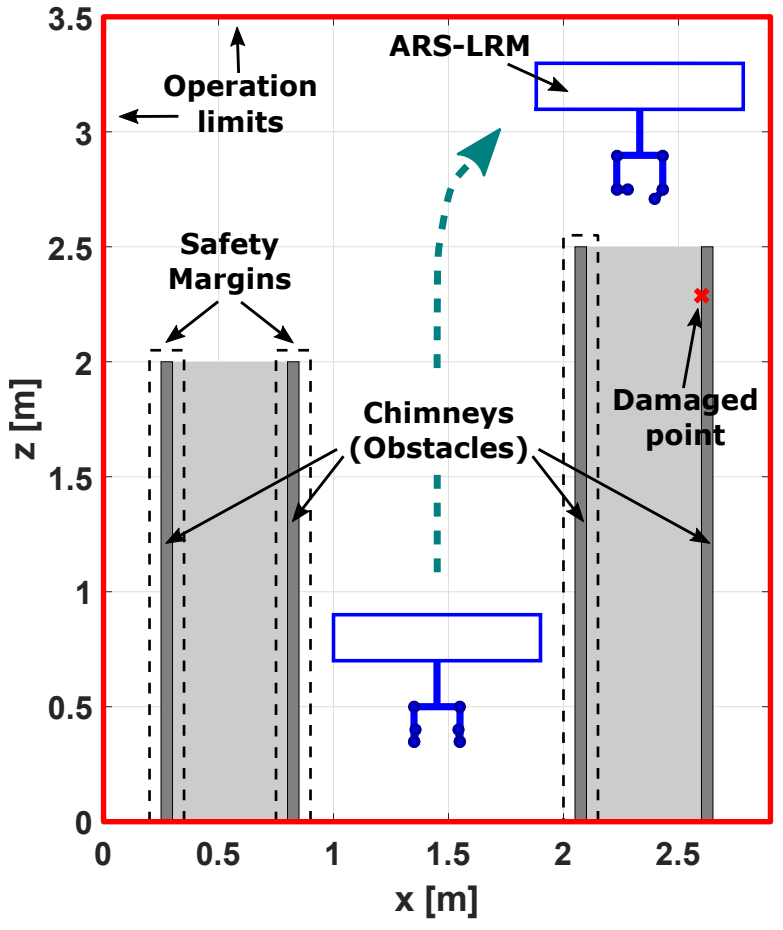

Fig. 13. Application scenario given by a chimney repairing task.

algorithm has been tested in a realistic industrial scenario given by a chimney repairing task. The schematic description of the scenario is shown in Fig. 13, where there are two chimneys represented by four dark grey rectangles. The two light grey rectangles corresponds to the free space inside the chimneys and the dotted surrounding areas denote safety regions whose violations will be treated as collisions. As depicted in the figure, the ARS-LRM system will be commanded to repair a crack inside the chimney (target point marked in red). Thanks to the bar extension of the ARS-LRM system, the aerial platform can operate out of the chimney with certain separation distance while the repairing task is being performed. This contributes to reduce the undesired aerodynamic effects that can be present both inside the chimney as well as in its surrounding space.

In order to perform the repairing operation, the ARS-LRM system has to reach an observation position over the right chimney. For this manoeuvre, the RRT*-DA algorithm was employed in [11] with satisfactory performance in terms of energy efficiency and collision avoidance. However, these results can be improved as far as time efficiency is concerned. The latter makes this scenario suitable to address a first proof of concept for the RRT*-DAVA algorithm presented in Sec. III-C.

\section{SIMULATION RESULTS}

This section analyses the results corresponding to the application of the RRT*-DAVA algorithm in the scenario previously described. The simulations have been organised around two main lines that endorse the need to employ the RRT*-DAVA algorithm for generating time-efficient trajectories in cluttered environments. On the one hand, the 
performance of the RRT*-DA algorithm has been evaluated, bringing to light the limitations of the method. On the other hand, a detailed analysis of the RRT*-DAVA performance in terms of time efficiency has been carried out.

In general terms, the simulations have been performed as follows. Firstly, the RRT*-DA algorithm as well as the more advanced RRT*-DAVA motion planner of the ARS-LRM system have been executed for the scenario under study. Then, the resultant plans (represented in the figures with light green lines for the RRT*-DA algorithm and with light orange lines for the RRT*-DAVA algorithm) have also been provided to the controlled ARS-LRM system in order to analyse the close-loop behaviour of the system when following the planned trajectories (represented in the figures with dark green lines for the RRT*-DA algorithm and with dark orange lines for the RRT*-DAVA algorithm).

The simulation work has been carried out in a MatlabSimulink framework that provides the graphical evolution of the system variables as well as the corresponding virtual reality animations. Both graphical outputs will be used throughout this section to illustrate the obtained results. Additionally, intuitive snapshot diagrams have also been included. In these diagrams, the dotted grey lines represent the complete simulated movement of the multirotor center of mass. Note that, these lines have a density of points inversely proportional to the velocity of the ARS-LRM system. On the other hand, the snapshots only cover some intermediate configurations to intuitively illustrate the qualitative behaviour of the system. These representative configurations are timeordered by identifying labels that go from the initial position (label 0) to the final position (label $f$ ) passing through a set of intermediate shadowed positions (labels $i_{1}, \ldots, i_{n}$,).

\section{A. Results Using the RRT*-DA Algorithm}

Fig. 14 and 15 present the results using the RRT*-DA method. As can be seen, this motion planner leads the ARS-LRM system to energy-efficient and collision-free trajectories thanks to both the RRT* optimal approach and the dynamics awareness. More particularly, these figures show how the planner generates an efficient trajectory with a reduced number of ARS-LRM movements while the feasibility of the executed trajectory -in terms of collision avoidance- is assured. A complete animation of the simulation results using the RRT*-DA algorithm as motion planner can be found in [14].

While previous results are satisfactory in terms of safety and energy efficiency, the time efficiency of the trajectory still admits some improvement. As was introduced in Sec. III-C, the application of the RRT*-DA method leads to constant velocity profiles in the resulting planned trajectories (see Fig. 16). This approach does not offer time-optimal results in non-homogeneously cluttered environments since those constant velocity profiles will end up equalling the lowest velocities required to safely avoid obstacles in the most cluttered areas (the north-east area in the chimney scenario). Therefore, while the RRT*-DA algorithm is properly commanding a lower velocity in the north-east area previously

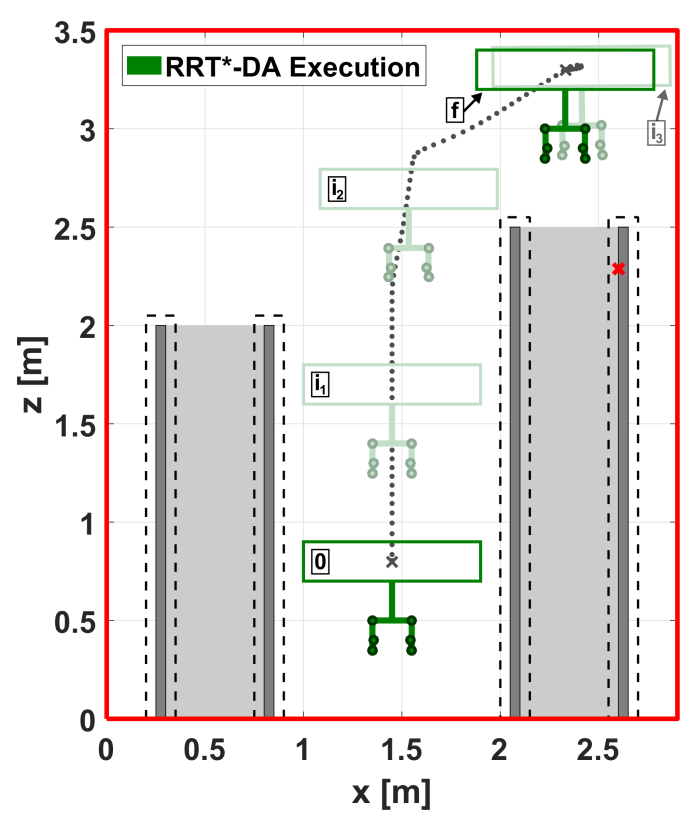

Fig. 14. Trajectory planned with the RRT*-DA algorithm and executed by the controlled ARS-LRM system.

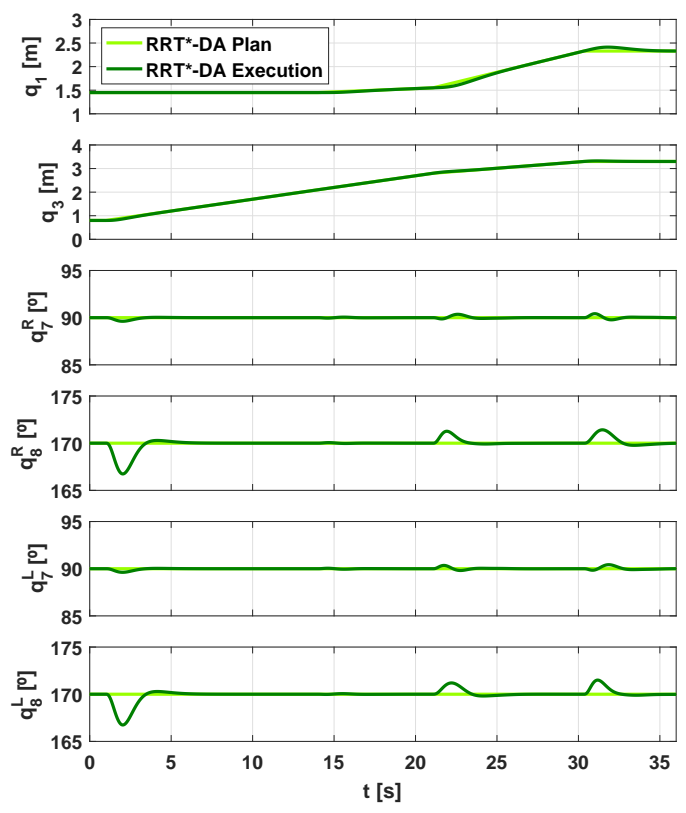

Fig. 15. Trajectory planned with the RRT*-DA algorithm (light green) and executed by the controlled ARS-LRM system (dark green).

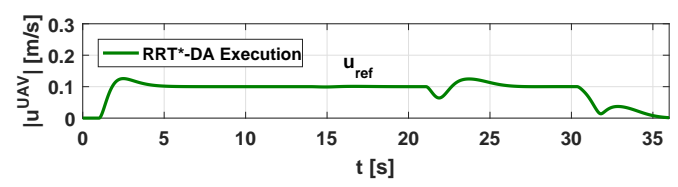

Fig. 16. Trajectory planned with the RRT*-DA algorithm and executed by the controlled ARS-LRM system. Evolution of the UAV velocity.

mentioned to guarantee safe operation, the algorithm is not seizing the opportunity to impose higher velocities in the area between the 2 chimneys taking advantage of the absence of constraints in this region. 


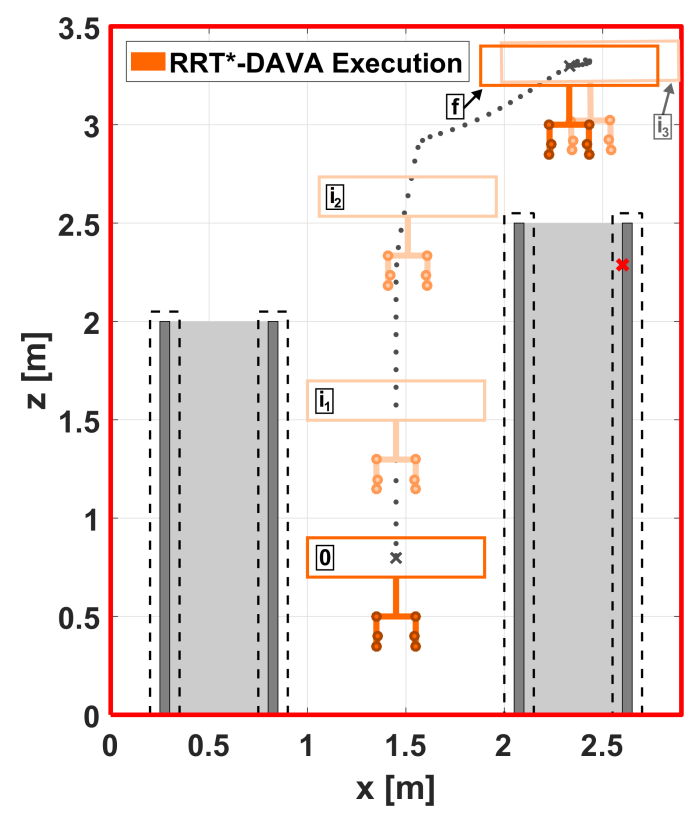

Fig. 17. Trajectory planned with the RRT*-DAVA algorithm and executed by the controlled ARS-LRM system.
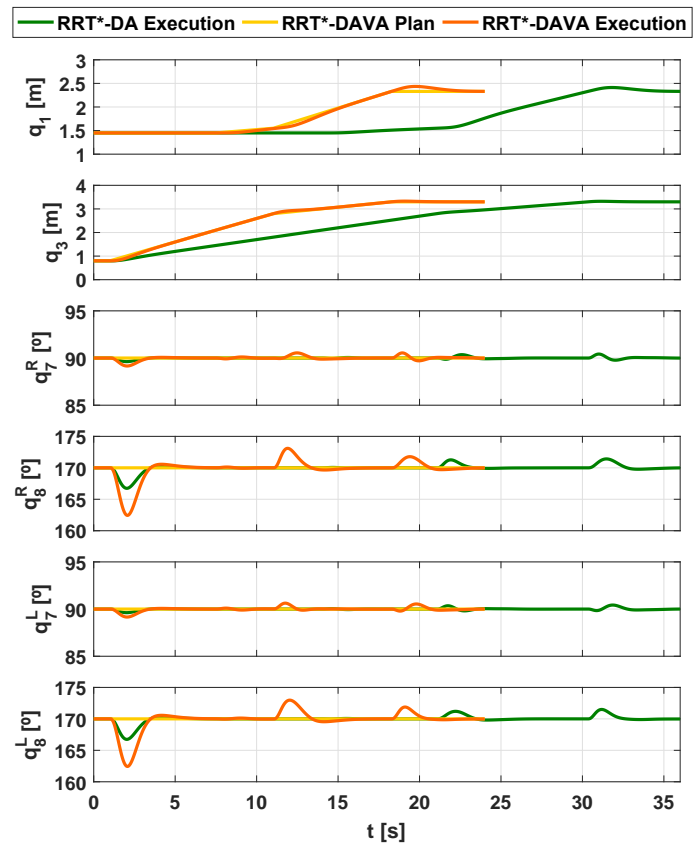

Fig. 18. Trajectory planned with the RRT*-DAVA algorithm (light orange) and executed by the controlled ARS-LRM system (dark orange). For comparison purposes, the dark green line represents the execution by the controlled ARS-LRM system of the trajectory planned with the RRT*-DA algorithm.

\section{B. Results Using the RRT*-DAVA Algorithm}

Previous subsection motivated the need to consider Velocity Adaptation within the motion planning problem when operating in cluttered environments. In order to deal with the above, the RRT*-DAVA algorithm has been applied.

Fig. 17 and 18 show the simulation results in the same manner as Fig. 14 and 15 do for the RRT*-DA algorithm. In view of these results, all the advantages of the

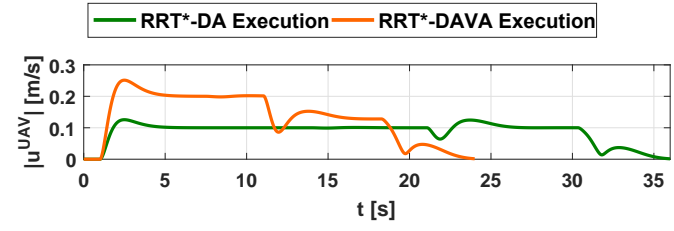

Fig. 19. Trajectory planned with the RRT*-DAVA algorithm and executed by the controlled ARS-LRM system. Evolution of the UAV velocity. For comparison purposes, the dark green line represents the execution by the controlled ARS-LRM system of the trajectory planned with the RRT*-DA algorithm.

RRT*-DA algorithm have been maintained. Moreover, with the RRT*-DAVA algorithm now it is possible to reach the goal state in $12 s$ less (a reduction of $33.33 \%$ in time). To better illustrate this reduction in execution time, Fig. 19 shows the evolution of the UAV velocity. According to the figure, the ARS-LRM system navigates at maximum reference velocity $u_{r e f}^{\max }=0.2 \mathrm{~m} / \mathrm{s}$ except when a reduction of velocity is required, for instance, in order to avoid a collision with the right part of the red limit. A complete animation of the simulation results using the RRT*-DAVA algorithm can be found in [15].

\section{CONCLUSIONS}

This paper extends previous works of the authors [7][11] in the field of motion planning strategies for aerial robotic systems. The most relevant contribution is the inclusion of Velocity Adaptation (VA) in the motion planner derived for an aerial robotic system with two arms for long-reach manipulation (ARS-LRM). This feature allows the consideration of the system velocity within the planning process but without increasing significantly the computational burden since there is not any extension in the planning space. This Velocity Adaptation approach allows a better optimization of the expected execution time of the planned trajectories in non homogeneously cluttered environments.

The validation of the presented motion planning strategy has been addressed by testing the RRT*-DAVA algorithm in a realistic industrial scenario, a chimney repairing task. As was discussed in the simulation section, the planned trajectories by RRT*-DAVA succeed in commanding efficiently -in terms of energy- the controlled ARS-LRM system without producing collisions with the obstacles existing in the scenario. Furthermore, the resulting trajectory from RRT*-DAVA clearly outperforms the RRT*-DA results in terms of time efficiency with a reduction of $33.33 \%$ in the time required to reach the goal state. These results endorse the validity of the approach proposed in this paper.

Regarding future extensions of the work, the presented results will be completed with a three-dimensional characterization of the ARS-LRM system. Then, experimental validation will be carried out by extending the platform prototype presented in [4] to integrate the long bar extension required for the ARS-LRM system. Finally, due to the relevance of aerodynamic effects such as the disturbances that may appear when an aerial platform operates close to other surfaces in 
cluttered environments, the motion planning strategies will be also extended to account for such aerodynamic effects.

\section{ACKNOWLEDGEMENTS}

This work has been supported by the European Projects AEROARMS and HYFLIERS, funded by the Horizon 2020 research and innovation programme of the European Commission under grant agreements No 644271 and 779411 respectively, and by the AEROMAIN project of the Spanish RETOS Programme DPI2014-59383-C2-1-R.

\section{REFERENCES}

[1] D. Mellinger, Q. Lindsey, M. Shomin, and V. Kumar, "Design, modeling, estimation and control for aerial grasping and manipulation," in Intelligent Robots and Systems (IROS), 2011 IEEE/RSJ International Conference on. IEEE, 2011, pp. 2668-2673.

[2] K. Kondak, F. Huber, M. Schwarzbach, M. Laiacker, D. Sommer, M. Bejar, and A. Ollero, "Aerial manipulation robot composed of an autonomous helicopter and a 7 degrees of freedom industrial manipulator," in Robotics and Automation (ICRA), 2014 IEEE International Conference on. IEEE, 2014, pp. 2107-2112.

[3] C. Korpela, M. Orsag, and P. Oh, "Towards valve turning using a dualarm aerial manipulator," in Intelligent Robots and Systems (IROS), 2014 IEEE/RSJ International Conference on. IEEE, 2014, pp. 34113416.

[4] A. Suarez, A. E. Jimenez-Cano, V. Vega, G. Heredia, A. RodriguezCastao, and A. Ollero, "Lightweight and human-size dual arm aerial manipulator," in Unmanned Aircraft Systems (ICUAS), 2017 International Conference on. IEEE, 2017, pp. 1778-1784.

[5] B. Yüksel, G. Buondonno, and A. Franchi, "Differential flatness and control of protocentric aerial manipulators with any number of arms and mixed rigid-/elastic-joints," in Intelligent Robots and Systems (IROS), 2016 IEEE/RSJ International Conference on. IEEE, 2016, pp. 561-566.
[6] R. Ragel, I. Maza, F. Caballero, and A. Ollero, "Comparison of motion planning techniques for a multi-rotor UAS equipped with a multijoint manipulator arm," in Research, Education and Development of Unmanned Aerial Systems (RED-UAS), 2015 Workshop on. IEEE, 2015, pp. 133-141.

[7] A. Caballero, M. Bejar, A. Rodriguez-Castaño, and A. Ollero, "Motion planning for long reach manipulation in aerial robotic systems with two arms," in 2017 European Conference on Mobile Robots (ECMR), 2017, pp. $1-7$.

[8] S. M. LaValle and J. J. Kuffner Jr, "Randomized kinodynamic planning," The International Journal of Robotics Research, vol. 20, no. 5 , pp. 378-400, 2001.

[9] E. Koyuncu and G. Inalhan, "A probabilistic B-spline motion planning algorithm for unmanned helicopters flying in dense 3D environments," in Intelligent Robots and Systems (IROS), 2008 IEEE/RSJ International Conference on. IEEE, 2008, pp. 815-821.

[10] C. Richter, A. Bry, and N. Roy, "Polynomial trajectory planning for aggressive quadrotor flight in dense indoor environments," in Robotics Research. Springer, 2016, pp. 649-666.

[11] A. Caballero, M. Bejar, A. Rodriguez-Castaño, and A. Ollero, "Motion planning with dynamics awareness for long reach manipulation in aerial robotic systems with two arms," The International Journal of Advanced Robotic Systems, 2018, submitted. [Online]. Available: https://hdvirtual.us.es/discovirt/index.php/s/wCK43aNHUlhVCO1

[12] K. Kondak, M. Bernard, N. Meyer, and G. Hommel, "Autonomously flying VTOL-robots: Modeling and control," in Robotics and Automation (ICRA), 2007 IEEE International Conference on. IEEE, 2007, pp. $736-741$

[13] Motiongenesis Kane 5.x. [Online]. Available: http://www.motiongenesis.com/

[14] Simulation results using the RRT*-DA algorithm. https://youtu.be/IZaDA0Dhx9c.

[15] Simulation results using the RRT*-DAVA algorithm https://youtu.be/LyBNchruV04. 